\title{
Recurrent Childhood Optic Nerve Astrocytoma
}

National Cancer Institute

\section{Source}

National Cancer Institute. Recurrent Childhood Optic Nerve Astrocytoma. NCI

Thesaurus. Code C7532.

The reemergence of optic nerve astrocytoma in childhood after a period of remission. 